\title{
Intervención de tres estrategias educativas para cursos de programación en educación superior
}

\section{Intervention of Three Educational Strategies for Higher Education Programming Courses}

\section{Arturo Rojas-López}

División de Tecnologías de la Información y Comunicación, Universidad Tecnológica de Puebla

Antiguo camino a la resurrección 1002-A, Zona Industrial Oriente, 72300 Puebla, México, (+52)2225134241, arturo.rojas@utpuebla.edu.mx

\section{Resumen}

El objetivo principal del presente trabajo es mostrar los resultados de intervenciones realizadas frente a grupo de tres estrategias educativas que permitieron tener mejores porcentajes de acreditación y calificación, así como disminución de deserción en comparación con los obtenidos en los últimos 8 años en los cursos iniciales de programación en la Universidad Tecnológica de Puebla. La primera intervención involucró la evaluación del pensamiento computacional a través de las habilidades de generalización, descomposición, abstracción, evaluación y diseño algorítmico; dicha evaluación fue la primera estrategia que permitió ofrecer a los estudiantes 10 escenarios de aprendizaje para el curso de Metodología de la programación. En la segunda intervención, 4 elementos fueron manipulados para ofrecer opciones de estudio en el curso de Programación, los cuales fueron contenidos, modos de trabajo, ritmos y tiempo y evaluación; fue la segunda estrategia con la intención de crear educación personalizada. En ambas intervenciones, el uso de la plataforma Moodle permitió exponer contenidos de aprendizaje y tener una herramienta extra para los estudiantes; la tercera estrategia fue en consecuencia el uso del b-learning. El principal resultado obtenido a través de encuestas voluntarias realizadas por los estudiantes fue la generación de una experiencia de aprendizaje que contribuyó a la motivación del estudiante en sintonía con las metas académicas de los cursos mencionados, por lo anterior se puede concluir que la combinación de las estrategias realizadas en las dos intervenciones mejoró los índices de acreditación y disminuyó el porcentaje de deserción, aunque aún hay trabajo por hacer para mejorar la calificación promedio.

\section{Palabras Clave}

Pensamiento computacional; Educación personalizada; b-learning; Educación superior; Estudiantes; Enseñanza de programación
Abstract

The main objective of present work is to show the results of interventions carried out in front of a group of three educational strategies that allowed having better percentages of accreditation and qualification, as well as decrease of dropout compared to those obtained in the last 8 years in initial courses of programming at the Technological University of Puebla. The firstintervention involved evaluation of computational thinking through skills of generalization, decomposition, abstraction, evaluation and algorithmic design, this evaluation was the first strategy that allowed offering students 10 learning scenarios for Programming Methodology course. In the second intervention, 4 elements were manipulated to offer study options in Programming course, which were content, work modes, rhythms and time and evaluation; it was the second strategy with intention of creating personalized education. In both interventions, use of Moodle platform allowed exposing learning content and having an extra tool for students; the third strategy was consequently the use of b-learning. The main result obtained through voluntary surveys carried out by students, was the generation of a learning experience that contributed to motivation of student in line with academic goals of the aforementioned courses, so it can be concluded that combination of the strategies carried out in the two interventions improved accreditation rates and decreased percentage of dropouts, although there is still work to be done to improve average rating

\section{Keywords}

Computational Thinking; Personalized Education; b-learning; Higher Education; Students; Programming Teaching 


\section{Introducción}

A partir del concepto de educación personalizada, ofrecido por Bernardo et al. (2011), como una concepción educativa ajena a un modo de entender la educación enmarcado en alguna corriente filosófica, psicológica o pedagógica concreta, sino que está abierta a todas las corrientes razonables de pensamiento que contribuyen a la formación de toda la persona; las intervenciones realizadas buscaron atender a lo que los estudiantes tienen en común, y lo que tienen de propio dentro del contexto académico de los cursos mencionados. A través de la plataforma Moodle y la oferta de opciones se pretendió estimular a cada estudiante para que vaya perfeccionado libre y responsablemente la capacidad de dirigir su propio conocimiento, cambiar de una educación tradicional hacia una personalizada, no es una estrategia aislada, así lo busca también el trabajo de Hart (2016), que propone una "adaptación de la educación considerando las características específicas de cada estudiante". El trabajo de investigación de Sadovaya et al. (2016) comenta que "es necesario desarrollar un nuevo modelo de estrategias educativas basadas en los principios de la personificación, el diálogo, la subjetividad, el enfoque individual y la complementariedad". Tekin et al. (2015) enmarcan su investigación dentro de los sistemas educativos basados en la Web, destacando su uso como un complemento "y, en algunos casos, en alternativas viables a la enseñanza tradicional en el aula"; en comparación a los MOOC resalta la observación de que "continúan siendo de una sola talla para todos", así propone un "método sistemático para diseñar un sistema educativo personalizado basado en la Web", destaca el trabajo anterior al comentar tres desafíos al momento de personalizar la educación, tales desafíos son: "(i) los estudiantes deben recibir enseñanza y capacitación personalizada según sus contextos (por ejemplo, clases ya tomadas, métodos de aprendizaje preferidos, etc.), (ii) para cada contexto específico, el mejor método de enseñanza y capacitación (por ejemplo, el tipo y orden de los materiales de enseñanza que se mostrarán) debe ser aprendido, (iii) la enseñanza y la capacitación deben adaptarse en línea, en función de los puntajes / retroalimentación (por ejemplo, pruebas, cuestionarios, examen final, gustos / disgustos, etc.) de los estudiantes”. El trabajo de Laksitowening y Hasibuan (2015) aporta también otros componentes que "juegan un papel importante en la personalización: estructura de aprendizaje integrada, modelo de aprendizaje, escenario de aprendizaje personalizado, selección de contenido personalizado y evaluación basada en evidencias" que les permitió proponer una arquitectura de e-learning personalizada que cumple con los estándares que hacen evidente el aprendizaje de competencias. Otro enfoque a considerar es el trabajo de Tejeda et al. (2015) donde a partir de las metas educativas del proceso de Bolonia, se presenta un "sistema de recomendación para proporcionar actividades personalizadas a los estudiantes para reforzar su educación individualizada" y sirve como ayuda a los profesores para proporcionar a los estudiantes un seguimiento personalizado de sus estudios. En el ámbito de las 
Tecnologías Móviles Personales, Kucirkova y Littleton (2017) argumentan que para integrarse en las escuelas, estas deben integrar la "noción de que el aprendizaje de los niños necesita ser adaptado a las aspiraciones de los individuos (es decir, personalizado) y participativo, implicando la consideración de múltiples perspectivas (es decir, pluralizadas)"; así, enfatizando el papel vital que desempeñan los educadores, comenta que "la personalización y pluralización deben conceptualizarse como fuerzas complementarias dentro de la reforma educativa del siglo XXI". El nuevo modelo educativo para la educación obligatoria, titulado “Educación para la libertad y creatividad en México" (2017), propone elementos de adaptación y flexibilidad que pueden ser considerados dentro de la educación personalizada: (i) Un método humanista, permitiendo adaptar el contenido a necesidades específicas y contextos de los estudiantes. (ii) La escuela debe enfocarse en alcanzar el máximo aprendizaje de todos sus estudiantes. (iii) El profesor debe generar entornos de aprendizaje inclusivos y es capaz de adaptar la curricular a su contexto específico. (iv) El sistema educativo es para todos los estudiantes y debe proporcionar las bases a cada uno para tener oportunidades, reconociendo su contexto social y cultural; por lo tanto la educación superior en México no está exenta de aspirar a la implementación de la educación personalizada, pero es una acción que no puede aplicarse en las clases cara a cara tradicionales donde los estudiantes no pueden ser tratados individualmente como lo expone Kostalányová (2017), sino que debe auxiliarse el profesor en tecnologías Web o e-learning. Así, se tiene un caso de éxito reportado por Zhao (2016) en Ingeniería Química donde propone un Método de Tres Etapas para mejorar la educación personalizada a lo largo de la carrera, no solo en una asignatura. Un caso similar, pues se enfoca en la enseñanza del lenguaje de programación 'C', es el trabajo de Chrysafiadi y Virvou (2015a), que reporta la identificación de las necesidades individuales de cada estudiante para completar el programa de entrenamiento en su propio ritmo y habilidades de aprendizaje, a lo que denomina el modelo del estudiante, que a la par del trabajo de Chrysafiadi y Virvou (2015b) detalla qué modelar, cómo y por qué. Otros trabajos de investigación que contribuyen al uso de la educación personalizada son Sun et al. (2016) y Morrowy et al. (2016); en el primero se proponen cinco actividades de aprendizaje de visualización aplicadas al aprendizaje de conceptos geométricos de matemáticas elementales con el objetivo de cultivar las habilidades del alumno en el aprendizaje independiente; en el segundo se proponen decisiones algorítmicas que faciliten la recomendación de los horarios de cursos personalizados según los antecedentes y los intereses de un alumno dado. Finalmente, asumiendo la importancia de la educación personalizada, Villegas et al. (2017) desarrolla técnicas de minería de datos aplicadas a los sistemas de gestión del aprendizaje, particularmente Moodle, para proporcionar información útil a los profesores en el objetivo de ofrecer educación adaptada a las necesidades de los estudiantes.

El pensamiento computacional (Wing, 2006, García-Peñalvo, 2016a y García-Peñalvo y Cruz-Benito, 2016) representa una propuesta adecuada para fomentar a temprana edad el aprendizaje de 
habilidades que beneficien a los estudiantes que ingresan en las áreas de Ciencia, Tecnología, Ingeniería y Matemáticas (STEM) (TACCLE 3 Consortium, 2017, García-Peñalvo, 2016b, García-Peñalvo, 2016c, García-Peñalvo, et al., 2016), la organización de torneos abiertos para niños de hasta 14 años indica una confianza global por parte de las instituciones educativas (UK Bebras Computational Thinking Challenge, 2015, Talent Search, 2015) y su evaluación es un tema de investigación que ha permitido la creación de pruebas (Román, et al., 2015), vinculación con el aprendizaje de la programación y la taxonomía de Bloom (Selby, 2015), y ha servido de forma efectiva para determinar escenarios de aprendizaje (Rojas-López y García-Peñalvo, 2016b). Particularmente, existen dos trabajos realizados por Czerkawski et al. (2015) y Weese (2016). El primero se propone revisar el estado actual del campo en educación superior y discutir si las habilidades del pensamiento computacional son relevantes fuera de los campos STEM. El segundo confirma el interés en el pensamiento computacional para el desarrollo curricular universitario de programación visual. Así, el trabajo de la aplicación del pensamiento computacional en la educación superior sigue siendo fuente de investigación para diferentes aristas educativas no exclusivas de las ciencias de la computación.

La mediana del porcentaje de deserción estudiantil al terminar el primer cuatrimestre septiembre - diciembre en la carrera de Tecnologías de la Información y Comunicación (TIC) de la Universidad Tecnológica de Puebla (UTP) del 2009 al 2016 es de 31,13. La mediana del porcentaje de acreditación estudiantil del curso Metodología de la programación en el periodo anterior es de 70,77. La mediana de calificación en el mismo curso y periodo de los estudiantes que acreditan es de 6,68. La mediana del porcentaje de deserción estudiantil al concluir el segundo cuatrimestre enero - abril del 2010 al 2017 es de 30,43. La mediana del porcentaje de acreditación estudiantil del curso Programación en el periodo anterior es de 71,89. La mediana de calificación en el mismo curso y periodo de los estudiantes que acreditan es de 7,03. Por lo anterior, se propusieron estrategias educativas durante los cuatrimestres de septiembre - diciembre de 2016 y enero - abril de 2017 para los cursos de Metodología de la programación y Programación respectivamente, con el objetivo de mejorar los indicadores de estudiantes acreditados en los cursos mencionados, la calificación promedio del grupo y reducir los porcentajes de deserción. Las intervenciones que se describen en el presente artículo se fundamentan en la evaluación del pensamiento computacional (PC) a los estudiantes de nuevo ingreso, particularmente las habilidades de Generalización, Descomposición, Abstracción, Diseño algorítmico y Evaluación; ofertar escenarios de aprendizaje con base al resultado de la evaluación del PC, el uso de la plataforma Moodle como herramienta para un entorno b-learning y cuatro elementos (Contenidos, Modos de trabajo, Ritmos y tiempos, y Evaluación) que permitieron ofrecer una educación personalizada.

El contenido del artículo está integrado de la siguiente forma. En la sección Contexto se describen las características de los grupos experimentales donde se aplicaron las estrategias y los grupos 
de control que sirvieron para comparar los resultados obtenidos de los cursos de Metodología de la programación y programación de los últimos 8 años. En la sección Descripción se explica el proceso de intervención y las estrategias educativas que se propusieron. En la sección Resultados se comentan los datos obtenidos al final de los cuatrimestres y su comparación con los datos de los años anteriores, así como resultados cualitativos de encuestas realizadas. Finalmente, en la sección de Conclusiones se comenta el impacto del trabajo realizado y el trabajo a futuro de la propuesta.

\section{Contexto}

Los grupos de control se caracterizan por ser clases presenciales en el aula o laboratorio, no utilizan la plataforma Moodle y realizan evaluaciones en dos momentos indicados por la respectiva academia que pueden ser a través de examen escrito, prácticas de laboratorio, tareas y proyecto final; por lo general, el profesor del curso puede optar por usar el lenguaje de programación Java o C\#. El curso Metodología de la programación se imparte a los alumnos de nuevo ingreso durante el primer cuatrimestre que corresponde al periodo septiembre - diciembre, para el presente trabajo los datos de acreditación, deserción y calificación promedio se obtuvieron del 2009 al 2016 dando un total de 3659 estudiantes. Los conocimientos generales del curso están indicados en la Tabla1. El curso de Programación se imparte a los alumnos del segundo cuatrimestre que corresponde al periodo enero abril, para el presente trabajo los datos (deserción, acreditación y calificación promedio) se obtuvieron del 2010 al 2017, dando un total de 2633 estudiantes. Los conocimientos generales del curso están indicados en la Tabla 2.

Los grupos experimentales correspondieron a los cursos donde me asignaron como profesor. Para el curso de Metodología de la programación se realizó la primera intervención en el $1^{\circ} \mathrm{C}$ y $1^{\circ} \mathrm{D}$ con 33 estudiantes en cada grupo. Para el curso de Programación la segunda intervención solo se aplicó al $2^{\circ} \mathrm{C}$ con 25 estudiantes. Para ambas intervenciones se elaboró material que se utilizó en la plataforma Moodle. La descripción del contenido en la plataforma es la siguiente. Nombre y objetivo de la asignatura encabezan el curso, unidades temáticas indicadas como etapas con su respectivo objetivo, y organizado por lecturas, audios, videos, actividades y evaluación. La Figura 1 muestra el aspecto del curso Metodología de la programación. 


\begin{tabular}{|c|l|}
\hline $\begin{array}{c}\text { Unidad } \\
\text { Temática }\end{array}$ & \multicolumn{1}{c|}{ Conocimientos } \\
\hline 1 & $\begin{array}{l}\text { Tipos de datos } \\
\text { Identificadores de variables }\end{array}$ \\
\hline 2 & $\begin{array}{l}\text { Operadores aritméticos } \\
\text { Operadores lógicos } \\
\text { Operadores relacionales } \\
\text { Jerarquía de operadores } \\
\text { Resolver expresiones }\end{array}$ \\
\hline 3 & $\begin{array}{l}\text { Uso de variable contador y acumulador } \\
\text { Estructura de selección (condicional) } \\
\text { Estructura de repetición (ciclo) } \\
\text { Diagrama de flujo } \\
\text { Diseño de algoritmos }\end{array}$ \\
\hline
\end{tabular}

\begin{tabular}{|c|l|}
\hline $\begin{array}{c}\text { Unidad } \\
\text { Temática }\end{array}$ & \multicolumn{1}{|c|}{ Conocimientos } \\
\hline 1 & $\begin{array}{l}\text { Fundamentos y características } \\
\text { de la Programación Orientada a } \\
\text { Objetos }\end{array}$ \\
\hline 2 & $\begin{array}{l}\text { Generalidades y características del } \\
\text { ambiente de desarrollo }\end{array}$ \\
\hline 3 & $\begin{array}{l}\text { Clases y Objetos } \\
\text { Estructuras de control } \\
\text { Encapsulamiento } \\
\text { Herencia } \\
\text { Polimorfismo }\end{array}$ \\
\hline 4 & Arreglos \\
\hline 5 & Manejo de excepciones \\
\hline
\end{tabular}

\section{Metodología de la Programación}

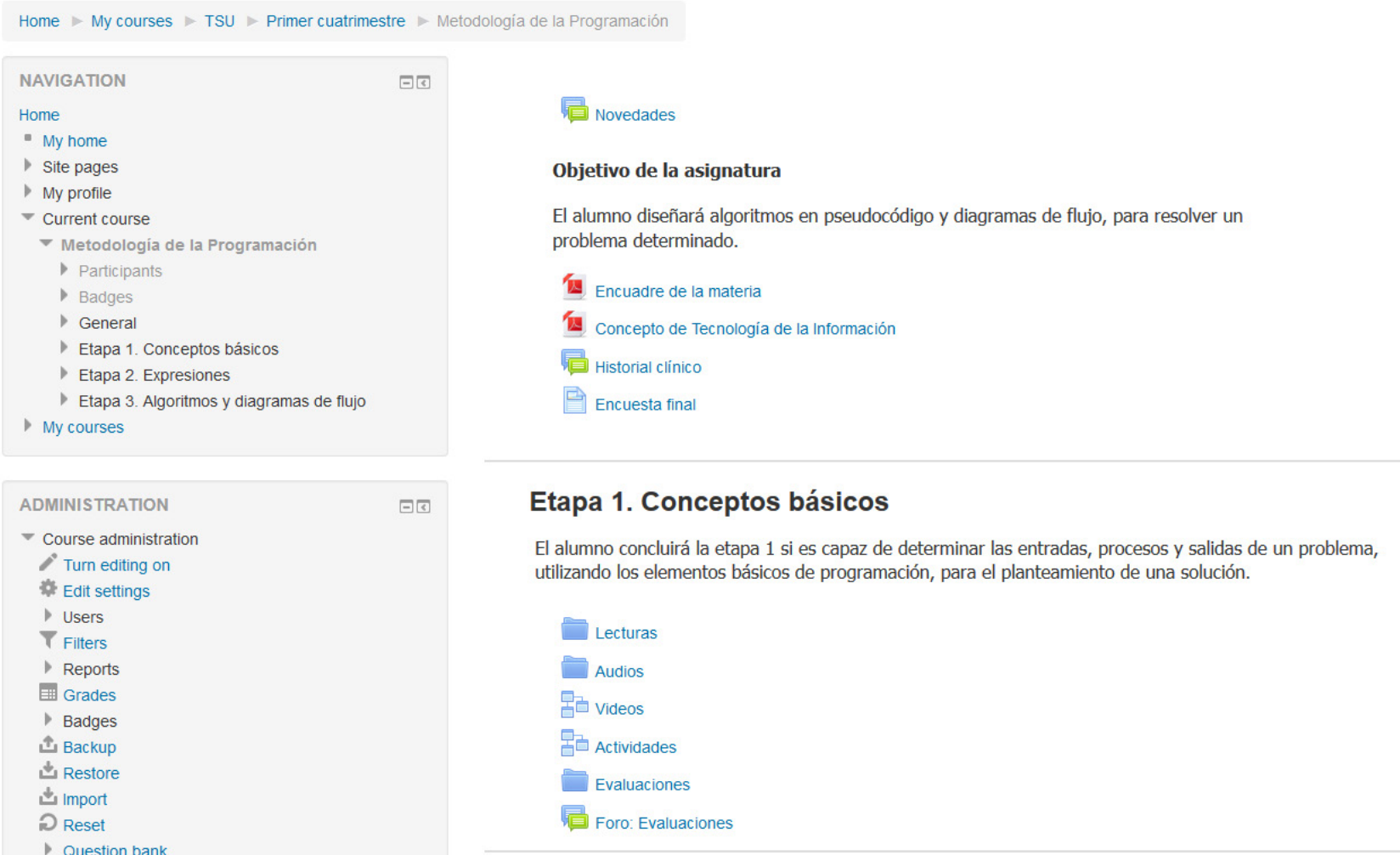




\section{Descripción}

Un elemento importante que se considera en la educación personalizada es la oferta de opciones a los estudiantes. La primera intervención fue desarrollada con las siguientes características. Evaluación del pensamiento computacional a través de las habilidades de Descomposición, Generalización, Abstracción, Diseño algorítmico y Evaluación, que se ajustaron bien a los temas del curso a través de la relación siguiente: cada tema tiene un objetivo de aprendizaje redactado con base a la taxonomía de Bloom y considerando el trabajo de Selby, cada habilidad del pensamiento computacional es mapeado a una categoría de Bloom, la Tabla 3 contiene la relación establecida de las unidades temáticas del curso Metodología de la Programación y las habilidades del pensamiento computacional. La evaluación permitió ofrecer 10 escenarios de aprendizaje usando tres modalidades de estudio: presencial, semi-presencial y en línea. En la modalidad presencial las clases se llevaron a cabo de forma tradicional, actividades prácticas y teóricas como lo indica el currículo de la asignatura en el horario asignado por la directora de la carrera. En la modalidad semi-presencial se aprovechó el uso de la plataforma Moodle para adaptar los conocimientos que debía aprender el estudiante según los reactivos correctos de la evaluación del pensamiento computacional, es en esta modalidad donde se dieron mayores opciones a los estudiantes y se organizaron días de asesorías. En la modalidad en línea el estudiante podía solicitar asesorías para aclarar dudas o entregar las evidencias de evaluación. Los ejercicios que se usaron para evaluar el PC y la descripción de los escenarios pueden encontrarse en Rojas-López y García-Peñalvo (2016a).

La segunda intervención fue desarrollada con las siguientes características. El estudiante tuvo la oportunidad de elegir los contenidos del curso, es decir, lecturas, audios o videos; tuvo la oportunidad de elegir la modalidad de aprendizaje (presencial, semi-presencial o en línea), los ritmos y tiempos de aprendizaje y evaluación, es decir, el estudiante se comprometió y determinó los momentos en que entregó los productos de evaluación y el tiempo que dedicó al estudio de los contenidos; finalmente tuvo la oportunidad de elegir las evidencias de evaluación que entregó tomando como guía una lista de cotejo por cada producto. 


\begin{tabular}{|l|l|l|l|}
\hline $\begin{array}{l}\text { Unidad } \\
\text { Temática }\end{array}$ & Verbos Usados & $\begin{array}{l}\text { Nivel taxonomía } \\
\text { Bloom }\end{array}$ & Habilidad PC \\
\hline $\begin{array}{l}\text { 1 Conceptos básicos, } \\
\text { 6 temas }\end{array}$ & $\begin{array}{l}\text { Saber: } \\
\text { Identificar, } \\
\text { Reconocer y } \\
\text { Describir }\end{array}$ & 2 Comprender & Abstracción \\
\cline { 2 - 3 } & $\begin{array}{l}\text { Saber Hacer: } \\
\text { Esquematizar y } \\
\text { Determinar }\end{array}$ & 4 Analizar & Descomposición \\
\hline $\begin{array}{l}\text { 2 Expresiones, } \\
\text { 3 temas }\end{array}$ & $\begin{array}{l}\text { Saber: } \\
\text { Identificar }\end{array}$ & 2 Comprender & Generalización \\
\cline { 2 - 3 } & $\begin{array}{l}\text { Saber Hacer: } \\
\text { Localizar, Resolver } \\
\text { y Convertir }\end{array}$ & 3 Aplicar & \\
\hline $\begin{array}{l}\text { 3 Algoritmos y } \\
\text { diagramas de flujo, } \\
\text { 4 temas }\end{array}$ & $\begin{array}{l}\text { Saber: } \\
\text { Reconocer, Identi- } \\
\text { ficar y Describir }\end{array}$ & 2 Comprender & Evaluación \\
\cline { 2 - 3 } $\begin{array}{l}\text { Saber Hacer: } \\
\text { Comparar y } \\
\text { Resolver }\end{array}$ & 5 Evaluar & Diseño algorítmico \\
\hline
\end{tabular}

Tabla 3. Relación de habilidades del PC y conocimientos del curso Metodología de la programación

\section{Resultados}

Para el cuatrimestre septiembre - diciembre de 2016, el porcentaje de deserción fue del 24,24 para el $1^{\circ} \mathrm{C}$ y del 27,27 para el $1^{\circ} \mathrm{D}$, que es menor en 6,89 y 3,86 por ciento respecto a la mediana de los últimos 8 años $(31,13)$; el porcentaje de acreditación del curso Metodología de la programación fue del 81,82 para el $1^{\circ} \mathrm{C}$ y del 72,73 para el $1^{\circ} \mathrm{D}$, que es mayor en 11,14 y 2,05 por ciento respecto a la mediana de los últimos 8 años (70,67); el promedio de calificación del mismo curso en los estudiantes del $1^{\circ} \mathrm{C}$ fue del 7,12 y del $1^{\circ} \mathrm{D}$ fue del 6,81 , mayor en 0,43 y 0,13 respecto a la mediana de los últimos 8 años $(6,68)$. La tabla 4 concentra la información comentada anteriormente. 


\begin{tabular}{|c|c|c|c|}
\hline \multirow{2}{*}{ Año } & \multicolumn{2}{|c|}{$\begin{array}{c}\text { Porcentajes } \\
\text { Primer cuatrimestre }\end{array}$} & $\begin{array}{c}\text { Promedio de } \\
\text { calificación }\end{array}$ \\
\cline { 2 - 3 } & Deserción & Acreditación & \\
\hline 2009 & 24,32 & 77,31 & 6,78 \\
\hline 2010 & 27,81 & 74,20 & 6,67 \\
\hline 2011 & 31,13 & 70,50 & 6,64 \\
\hline 2012 & 31,14 & 71,50 & 6,69 \\
\hline 2013 & 35,20 & 65,23 & 6,18 \\
\hline 2014 & 42,24 & 58,81 & 6,31 \\
\hline 2015 & 30,30 & 70,85 & 6,81 \\
\hline 2016 & 34,00 & 68,25 & 6,75 \\
\hline Mediana & 31,13 & 70,67 & 6,68 \\
\hline $1^{\circ} \mathrm{C} 2016$ & 24,24 & 81,82 & 7,12 \\
\hline $1^{\circ} \mathrm{D} 2016$ & 27,27 & 72,73 & 6,81 \\
\hline
\end{tabular}

Tabla 4. Información en los últimos 8 años primer cuatrimestre

Para el cuatrimestre enero - abril de 2017, el porcentaje de deserción fue del 8,0 para el $2^{\circ} \mathrm{C}$, que es menor en 22,76 por ciento respecto a la mediana de los últimos 8 años $(30,76)$; el porcentaje de acreditación del curso Programación fue del 92,0, que es mayor en 20,11 respecto a la mediana de los últimos 8 años $(71,89)$; el promedio de calificación del mismo curso en los estudiantes del $2^{\circ} \mathrm{C}$ fue de 7,09, mayor en 0,06 por ciento respecto a la mediana de los últimos 8 años $(7,03)$. La tabla 5 reúne la información anterior.

Se realizaron encuestas voluntarias en ambos cursos para conocer la aceptación de las estrategias de aprendizaje realizadas en las respectivas intervenciones; a continuación se presentan los resultados. Para el curso Metodología de la programación se preguntó si la modalidad de trabajo (presencial, semipresencial o en línea) le parecía al estudiante adecuada con su expectativa de aprendizaje, de los 66 estudiantes 24 contestaron la pregunta y el 75,0\% indicó que estaba de acuerdo; también se preguntó si la evaluación del pensamiento computacional, a través de las habilidades de Descomposición, Generalización, Abstracción, Diseño algorítmico y Evaluación, al inicio del cuatrimestre fue una actividad acertada para determinar el mejor entorno de aprendizaje, el 73,3\% de los 24 estudiantes respondieron que Sí. Para el curso de Programación se preguntó si el contenido del curso contribuyó a la formación profesional, de los 25 estudiantes solo 22 contestaron la encuesta voluntaria y el 100\% estuvo de acuerdo con el contenido del curso. 


\begin{tabular}{|l|c|c|c|}
\hline \multirow{2}{*}{ Año } & \multicolumn{2}{|c|}{$\begin{array}{c}\text { Porcentajes } \\
\text { Segundo cuatrimestre }\end{array}$} & $\begin{array}{c}\text { Promedio de } \\
\text { calificación }\end{array}$ \\
\cline { 2 - 3 } & Deserción & Acreditación & \\
\hline 2010 & 16,48 & 85,20 & 7,0 \\
\hline 2011 & 33,69 & 70,45 & 6,29 \\
\hline 2012 & 29,99 & 73,07 & 6,92 \\
\hline 2013 & 28,22 & 73,87 & 7,07 \\
\hline 2014 & 31,38 & 71,62 & 7,24 \\
\hline 2015 & 30,89 & 71,09 & 6,94 \\
\hline 2016 & 30,63 & 72,17 & 7,05 \\
\hline 2017 & 32,25 & 69,57 & 7,09 \\
\hline Mediana & 30,76 & 71,89 & 7,03 \\
\hline $2^{\circ} \mathrm{C} 2017$ & 8,0 & 92,0 & 7,09 \\
\hline
\end{tabular}

Tabla 5. Información en los últimos 8 años segundo cuatrimestre

\section{Conclusiones}

Las intervenciones realizadas implementando las estrategias descritas en el artículo: propuesta de un escenario de aprendizaje a partir de la evaluación del pensamiento computacional, ofrecer opciones de evidencias evaluativas, ritmo de aprendizaje y tiempo de entrega de productos determinados por los estudiantes, opciones en los formatos de los contenidos y diferentes modalidades de aprendizaje (presencial, semi-presencial y en línea) para ofrecer una educación personalizada, permitieron obtener un porcentaje de deserción menor a los registrados en los últimos 8 años al finalizar el primer y segundo cuatrimestre, así como un porcentaje de acreditación mayor en los cursos de Metodología de la programación y Programación en el mismo periodo. Respecto al promedio de la calificación en ambos cursos, no se registró un incremento significante, pero es una evidencia de que se ha mantenido la calidad evaluativa. A pesar de haber obtenido números favorables y que los estudiantes indicaron aceptación de las propuestas realizadas aún se deben trabajar las intervenciones para mejorar los resultados y tener más participantes al momento de contestar las encuestas; el trabajo a futuro incluye realizar un seguimiento puntual de las actividades en línea de los estudiantes, que permita mejorar la calificación promedio, pues como indican los resultados fue baja al igual que la mediana de los últimos 8 años y no representa un elemento favorable para la carreta de TIC en la UTP.

\section{Agradecimientos}

El presente trabajo es realizado dentro del programa de Doctorado en Educación en la Sociedad del Conocimiento de la Universidad de Salamanca (García-Peñalvo, 2013, 2014, 2015). 


\section{Referencias}

Bernardo, J., Javaloyes, J. J. y Calderero, J. F. (2011). Educación personalizada: principios, técnicas y recursos. Madríd: Síntesis.

Chrysafiadi, K. y Virvou, M. (2015a). A novel hybrid student model for personalized education. Advances in Personalized Web-Based Education (pp. 61-90). Cham: Springer. doi:http:// doi.org/10.1007/978-3-319-12895-5_3

Chrysafiadi, K. y Virvou, M. (2015b). Student Modeling for Personalized Education: A Review of the Literature. Advances in Personalized Web-Based Education (pp. 1-24). Cham: Springer. doi:http://doi.org/10.1007/978-3-319-12895-5_1

Czerkawski, B. C. y Lyman, E. W. (2015). Exploring Issues About Computational Thinking in Higher Education. TechTrends, 59(2), 57-65. doi:http://doi.org/10.1007/s11528-015-0840-3

García-Peñalvo, F. J. (2013). Education in knowledge society: A new PhD programme approach. In F. J. García-Peñalvo (Ed.), Proceedings of the First International Conference on Technological Ecosystems for Enhancing Multiculturality (TEEM'13) (Salamanca, Spain, November 14-15, 2013) (pp. 575-577). New York, NY, USA: ACM. doi:http://doi.org/10.1145/2536536.2536624.

García-Peñalvo, F. J. (2014). Formación en la sociedad del conocimiento, un programa de doctorado con una perspectiva interdisciplinar. Education in the Knowledge Society, 15(1), 4-9.

García-Peñalvo, F. J. (2015). Engineering contributions to a Knowledge Society multicultural perspective. IEEE Revista Iberoamericana de Tecnologías del Aprendizaje (IEEE RITA), 10(1), 17-18. doi:http://doi.org/10.1109/RITA.2015.2391371.

García-Peñalvo, F. J. (2016a). What Computational Thinking Is. Journal of Information Technology Research, 9(3), v-viii.

García-Peñalvo, F. J. (2016b). Proyecto TACCLE3 - Coding. In F. J. García-Peñalvo y J. A. Mendes (Eds.), XVIII Simposio Internacional de Informática Educativa, SIIE 2016 (pp. 187-189). Salamanca, España: Ediciones Universidad de Salamanca.

García-Peñalvo, F. J. (2016c) A brief introduction to TACCLE 3 - Coding European Project. In F. J. García-Peñalvo y J. A. Mendes (Eds.), 2016 International Symposium on Computers in Education (SIIE 16). USA: IEEE. doi:http://doi.org/10.1109/SIIE.2016.7751876.

García-Peñalvo, F. J. y Cruz-Benito, J. (2016). Computational thinking in pre-university education. In F. J. García-Peñalvo (Ed.), Proceedings of the Fourth International Conference on Technological Ecosystems 
for Enhancing Multiculturality (TEEM'16) (Salamanca, Spain, November 2-4, 2016) (pp. 13-17). New York, NY, USA: ACM. doi:http://doi.org/10.1145/3012430.3012490.

García-Peñalvo, F. J., Reimann, D., Tuul, M., Rees, A. y Jormanainen, I. (2016). An overview of the most relevant literature on coding and computational thinking with emphasis on the relevant issues for teachers. TACCLE3 Consortium. Belgium: TACCLE3 Consortium. doi:http://doi.org/10.5281/zenodo.165123

Hart, S. A. (2016). Precision Education Initiative: Moving Toward Personalized Education. Mind, Brain, and Education, 10(4), 209-211. doi:http://doi.org/10.1111/mbe.12109.

Kostolányová, K. (2017). Adaptation of Personalized Education in E-learning Environment. In T. T. Wu, R. Gennari, Y. M. Huang, H. Xie y Y. Cao (Eds.), Emerging Technologies for Education. SETE 2016 (pp. 433-442). Cham: Springer. doi:http://doi.org/10.1007/978-3-319-52836-6_46

Kucirkova, N. y Littleton, K. (2017). Developing personalised education for personal mobile technologies with the pluralisation agenda. Oxford Review of Education, 43(3), 276-288. doi:http://doi.org/10.1080/03054985.2017.1305046

Secretaría de Educación Pública. (2017). Ruta para la implementación del modelo educativo. SEPMéxico (pp. 14-16).

Laksitowening, K. A. y Hasibuan, Z. A. (2015). Personalized e-learning architecture in standardbased education. Proceedings - 2015 International Conference on Science in Information Technology: Big Data Spectrum for Future Information Economy, ICSITech 2015 (pp. 110-114). doi:http://doi.org/10.1109/ICSITech.2015.7407787.

Morrowy, T., Sarvestaniz, S. S. y Hursony, A. R. (2016). Algorithmic decision support for personalized education. Proceedings - 2016 IEEE 17th International Conference on Information Reuse and Integration, IRI 2016 (pp. 188-197). doi:http://doi.org/10.1109/IRI.2016.32

Román M., Pérez J. C. y Jiménez C. (2015). Test de Pensamiento Computacional: diseño y psicometría general Computational Thinking Test: design y general psychometry. III Congreso Internacional sobre Aprendizaje, Innovación y Competitividad (CINAIC 2015), octubre 14-16, 2015, Madrid, España.

Rojas-López, A. y García-Peñalvo, F. J. (2016a). Personalized contents based on cognitive level of student's computational thinking for learning basic competencies of programming using an environment b-learning. In F. J. García-Peñalvo (Ed.), Proceedings of the Fourth International Conference on Technological Ecosystems for Enhancing Multiculturality (TEEM'16) (Salamanca, Spain, November 2-4, 2016) (pp. 1139-1145). New York, NY, USA: ACM. doi:http://doi.org/10.1145/3012430.3012660

Rojas-López, A. y García-Peñalvo, F. J. (2016b). Relationship of knowledge to learn in programming 
methodology and evaluation of computational thinking. In F. J. García-Peñalvo (Ed.), Proceedings of the Fourth International Conference on Technological Ecosystems for Enhancing Multiculturality (TEEM'16) (Salamanca, Spain, November 2-4, 2016) (pp. 73-77). New York, NY, USA: ACM. doi: http://doi.org/10.1145/3012430.3012499

Sadovaya, V. V., Korshunova, O. V. y Nauruzbay, Z. Z. (2016). Personalized education strategies. Mathematics Education, 11(1), 199-209. doi:http://doi.org/10.12973/iser.2016.21019a

Selby, C. C. (2015). Relationships: computational thinking, pedagogy of programming, and Bloom's Taxonomy. In Proceedings of the Workshop in Primary and Secondary Computing Education (WiPSCE '15) (pp. 80-87). New York, NY, USA: ACM. doi:http://doi.org/10.1145/2818314.2818315.

Sun, N., Li, K. y Zhu, X. (2016). Action Research on Visualization Learning of Mathematical Concepts Under Personalized Education Idea: Take Learning of Geometrical Concepts of Elementary Math for Example. In S. Cheung, L. Kwok, J. Shang, A. Wang y R. Kwan(Eds.), Blended Learning: Aligning Theory with Practices. ICBL 2016 (pp. 348-359). Cham: Springer. doi:http://doi.org/10.1007/978-3-319-41165-1_31

TACCLE 3 Consortium. (2017) TACCLE 3: Coding Erasmus + Project website. Retrieved from http:// www.taccle3.eu/

Talent Search (2015). Elite: Grade 12+, Institute of IT Professionals South Africa, available http://www. olympiad.org.za.

Tejeda-Lorente, A., Bernabé-Moreno, J., Porcel, C., Galindo-Moreno, P. y Herrera-Viedma, E. A. (2015). Dynamic recommender system as reinforcement for personalized education by a fuzzly linguistic web system. Procedia Computer Science, 55, 1143-1150. doi:http://doi.org/10.1016/j.procs.2015.07.084

Tekin, C., Braun, J. y Van Der Schaar, M. (2015). eTutor: Online learning for personalized education. ICASSP, IEEE International Conference on Acoustics, Speech and Signal Processing - Proceedings, (pp. 5545-5549). EEUU: IEEE. doi:http://doi.org/10.1109/ICASSP.2015.7179032.

UK Bebras Computational Thinking Challenge, answers (2015). University of Oxford, available http:// www.bebras.org

Villegas-Ch, W. y Luján-Mora, S. (2017). Analysis of data mining techniques applied to LMS for personalized education. EDUNINE 2017 - IEEE World Engineering Education Conference: Engineering Education - Balancing Generalist and Specialist Formation in Technological Carriers: A Current Challenge, Proceedings (pp. 85-89). EEUU: IEEE. doi:http://doi.org/10.1109/EDUNINE.2017.7918188

Weese, J. L. (2016). Mixed methods for the assessment and incorporation of computational thinking in K-12 and higher education. ICER 2016 - Proceedings of the 2016 ACM Conference on International Computing Education Research (pp. 279-280). New York, NY, USA: ACM. 
doi: http://doi.org/10.1145/2960310.2960347

Wing J. M. (2006). Computational Thinking. Communications of the ACM, 49(3), 33-35. doi:http://doi.org/10.1145/1118178.1118215.

Zhao, F.-Q. (2016). Personalized Education Approaches for Chemical Engineering and Relevant Majors. MATEC Web of Conferences, 68, doi:http://doi.org/10.1051/matecconf/20166820003 